\title{
Sea blue histiocytosis: a common abnormality of the bone marrow in myelodysplastic syndromes
}

\author{
M R Howard, P J L Kesteven
}

\begin{abstract}
Aims-To determine whether myelodysplastic syndromes (MDS) are associated with sea blue histiocytosis in the bone marrow.
\end{abstract}

Methods-A retrospective review of bone marrow aspirates from 35 patients presenting consecutively with MDS and from 20 patients with each of the following: normal marrow appearance (routine staging for non-Hodgkin's lymphoma), polycythaemia rubra vera, immune thrombocytopenic purpura (ITP), chronic myeloid leukaemia (CML) in chronic phase.

Results-Sea blue histiocytes were present in the marrow in 12 of 35 cases of MDS and occurred in large numbers in three of these cases. Sea blue histiocytes varied in the degree of cytoplasmic granularity and some cells were intermediate in appearance between classic sea blue histiocytes and pseudo-Gaucher cells. Sea blue histiocyte granules additionally stained positively with Sudan black and periodic acid schiff. Sea blue histiocytes occurred only in the presence of marrow hypercellularity. Their presence did not correlate with FAB subtype, degree of dyserythropoeisis, or megakaryocyte numbers. No sea blue histiocytes were found in the normal marrow or polycythaemia cases. In ITP sea blue histiocytes were seen in two of 20 cases and in chronic myeloid leukaemia in eight of 20 cases.

Conclusions-Sea blue histiocytes are a common cytological feature in the bone marrow of patients with MDS. As other disorders frequently associated with marrow sea blue histiocytes are relatively rare MDS is probably the most common cause of this phenomenon in a northern European population.

(F Clin Pathol 1993;46:1030-1032)

Sea blue histiocytes were first described in the bone marrow by Wewalka in $1950 .{ }^{1}$ Typically these are large macrophages varying from 20 to $60 \mu \mathrm{m}$ in diameter with a single eccentric nucleus whose cytoplasm is packed with sea blue or blue green granules when stained with Wright-Giemsa. They may also occur in the spleen, liver, lymph nodes, and tonsils. ${ }^{2}$

In 1970 the Mayo Clinic group described the "sea blue histiocyte syndrome" as a new distinct clinical entity. ${ }^{3}$ Sea blue histiocytes, however, are more frequently seen in the marrow as a secondary phenomenon in association with a variety of metabolic and haematological disorders. They occasionally appear in the marrow in the absence of any overt disease. Haematological disorders reported to be associated with marrow sea blue histiocytes are chronic myeloid leukaemia, chronic immune thrombocytopenic purpura, sickle cell anaemia, thalassaemia, and polycythaemia rubra vera. ${ }^{2}$ With the exception of chronic myeloid leukaemia, ${ }^{4}$ this is confined to occasional case reports.

Heyhoe and Flemens, in their atlas of haematological cytology, note that sea blue histiocytes may be seen in "dysmyelopoietic and dyserythropoietic states". ${ }^{5}$ Kattlove et al, in reply to the initial report of sea blue histiocyte syndrome, argued that these cells may occur where there is increased turnover of blood or blood precursor cells. ${ }^{6}$ Haematological texts and review articles containing apparently exhaustive lists of the causes of marrow sea blue histiocytes ${ }^{2-11}$ do not refer to the myelodysplastic syndromes (MDS), perhaps because they have only recently been consolidated into an internationally recognised classification. ${ }^{12}$ The objective of this study was to determine whether myelodysplasia is associated with marrow sea blue histiocytosis and, if so, how frequently.

\section{Methods}

The patients studied represent all diagnosed cases of MDS presenting to the Haematology Department at the Freeman Hospital between January 1985 and September 1992. All had a bone marrow aspirate taken at presentation. Bone marrow aspirates were stained with May-Grünwald-Giemsa. Romanovsky stained bone marrow aspirates from all patients identified from departmental records were reexamined for this study and the diagnosis of MDS confirmed.

The total number of sea blue histiocytes in each aspirate smear was determined. Cases were divided into one of four groups: none; occasional (1-5 sea blue histiocytes); moderate (6-20); large (more than 20). Previously unstained aspirate smears from those with large numbers of sea blue histiocytes were stained with Sudan black and periodic acid schiff (PAS).

The following details of each patient were recorded: age (years); sex; FAB subtype of MDS; cellularity of bone marrow smear (decreased, normal, increased); degree of dyserythropoeisis (slight, moderate, great); number of megakaryocytes (decreased, normal, increased); number of sea blue histiocytes (as detailed). 


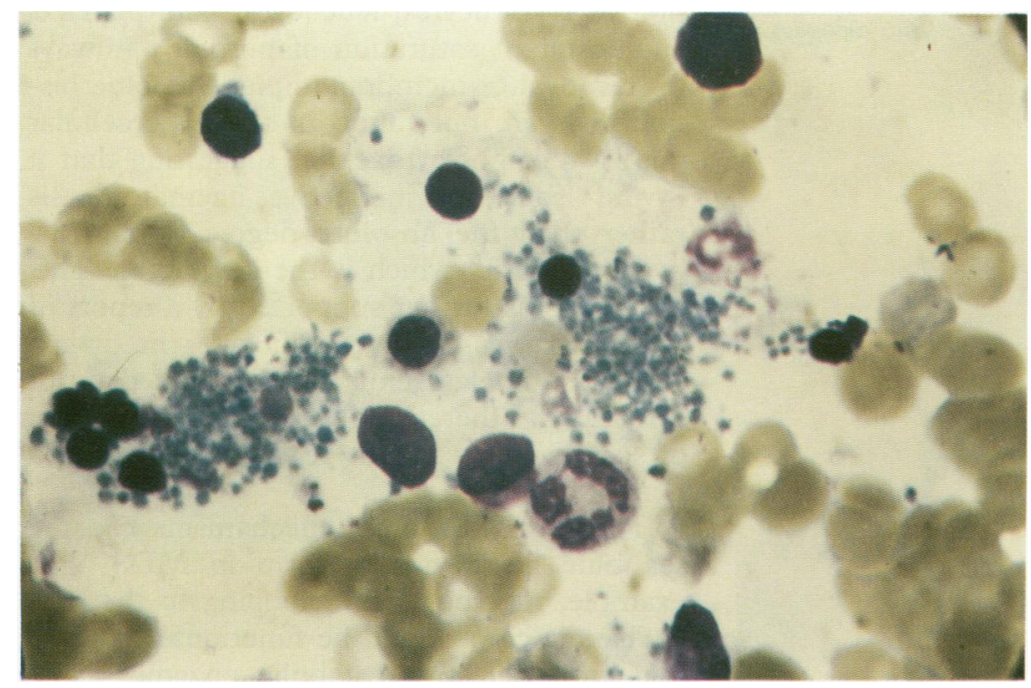

Figure 1 Heavily granulated sea blue histiocytes. Marrow aspirate from an 84 year old man with refractory anaemia with excess blasts (RAEB).

Bone marrow aspirates from the following groups of patients were also studied for sea blue histiocytes using identical methods:

1 "Normal" bone marrows (routine staging aspirate in patients with non-Hodgkin's lymphoma with no morphological abnormality detected).

2 Polycythaemia rubra vera.

3 Immune thrombocytopenic purpura (ITP).

4 Chronic myeloid leukaemia (CML) in chronic phase.

Each group comprised the 20 most recently presenting patients with the relevant diagnosis who had had a bone marrow aspirate performed. In each case the diagnosis was confirmed by review of patient notes and bone marrow morphology.

\section{Results}

MYELODYSPLASTIC SYNDROMES

Marrow aspirates from 35 patients with confirmed MDS were examined. There were 22 men and 13 women with a median age of 73

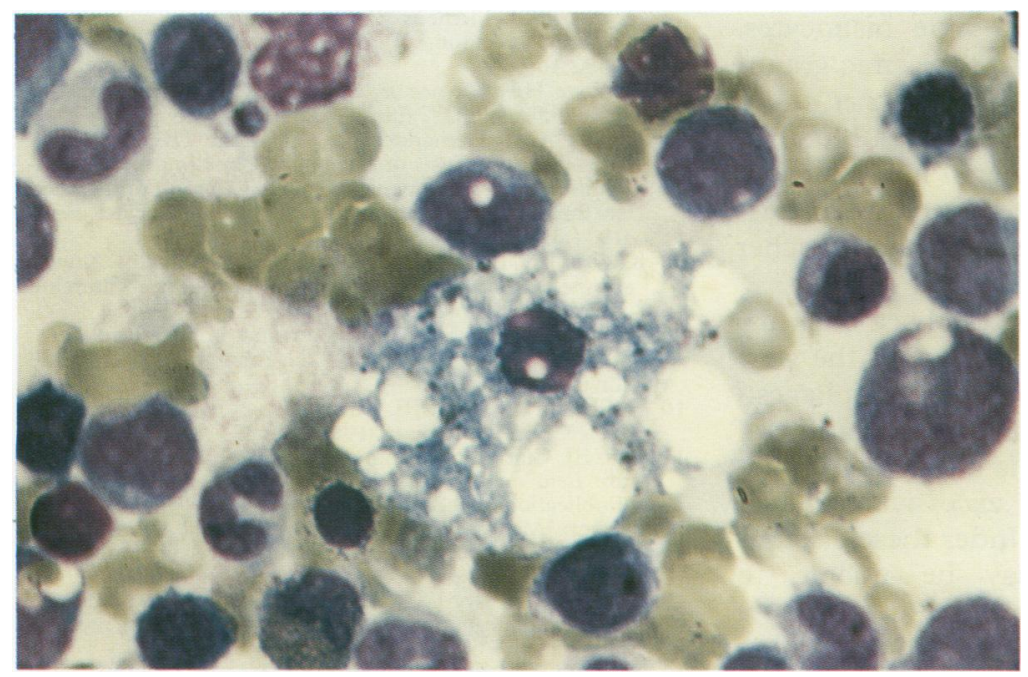

Figure 2 Sea blue histiocyte in the marrow of a 71 year old man with refractory anaemia with excess blasts in transformation (RAEB-t). Compared with the forms shown in figure 1, the cell has a less granular cytoplasm with vacuolation. years (range 48-88 years). FAB subtypes were as follows: refractory anaemia $n=12$; refractory anaemia with ring sideroblasts (RAS) $n=4$; refractory anaemia with excess blasts (RAEB) $\mathrm{n}=5$; refractory anaemia with excess blasts in transformation (RAEB-t) $\mathrm{n}=3$; chronic myelomonocytic leukaemia (CMML) $\mathrm{n}=11$.

Sea blue histiocytes were documented in 12 of 35 cases. The numbers of sea blue histiocytes were distributed as follows: occasional in seven cases (refractory anaemia $\mathrm{n}=3$, CMML $n=3$, RAEB $n=1$ ); moderate in two (RAEB-t, CMML); large in three (refractory anaemia, RAS, RAEB). In the three cases characterised by large numbers of sea blue histiocytes, the cytoplasmic granules stained positively for Sudan black and PAS.

All cases in which sea blue histiocytes occurred were characterised by hypercellularity of the marrow but this was also true for most cases in which they were absent (20/23). There was no correlation between the presence of sea blue histiocytes and FAB subtype, degree of dyserythropoeisis, or megakaryocyte numbers. Too few patients had been transfused before to assess the effect of blood transfusion on the numbers of sea blue histiocytes.

In most cases the appearance of sea blue histiocytes varied. Thus heavily granulated sea blue histiocytes (fig 1) frequently coexisted with more sparsely granulated forms (fig 2). Some cells showed features intermediate between sea blue histiocytes and pseudoGaucher cells (fig 3A).

\section{OTHER DIAGNOSES}

No sea blue histiocytes were present in the "normal" or polycythaemia rubra vera cases. In ITP moderate numbers of sea blue histiocytes were present in one case and occasional numbers in a second case. In CML they were present in eight out of 20 cases but only occurred in large numbers in one case. The sea blue histiocytes in CML frequently contained forms with features intermediate between classic sea blue histiocytes and pseudo-Gaucher cells.

\section{Discussion}

Sea blue histiocytes have been most often described in CML. The finding of sea blue histiocytes in eight of 20 cases of CML in this study is consistent with earlier reports. ${ }^{413}$ The presence of sea blue histiocytes in two patients with ITP confirms the observation of Rywlin et al, ${ }^{7}$ but suggests that they occur less frequently than in CML. A third of the patients with MDS had sea blue histiocytes in their marrow aspirates. It is not clear whether the failure of others to document this high prevalence reflects prior variations in the nomenclature of myelodysplasia or simple lack of observation. The former seems more likely. Sea blue histiocytes occurred only in the presence of marrow hypercellularity but two few hypocellular cases were examined to confirm a genuine correlation with cellularity. 

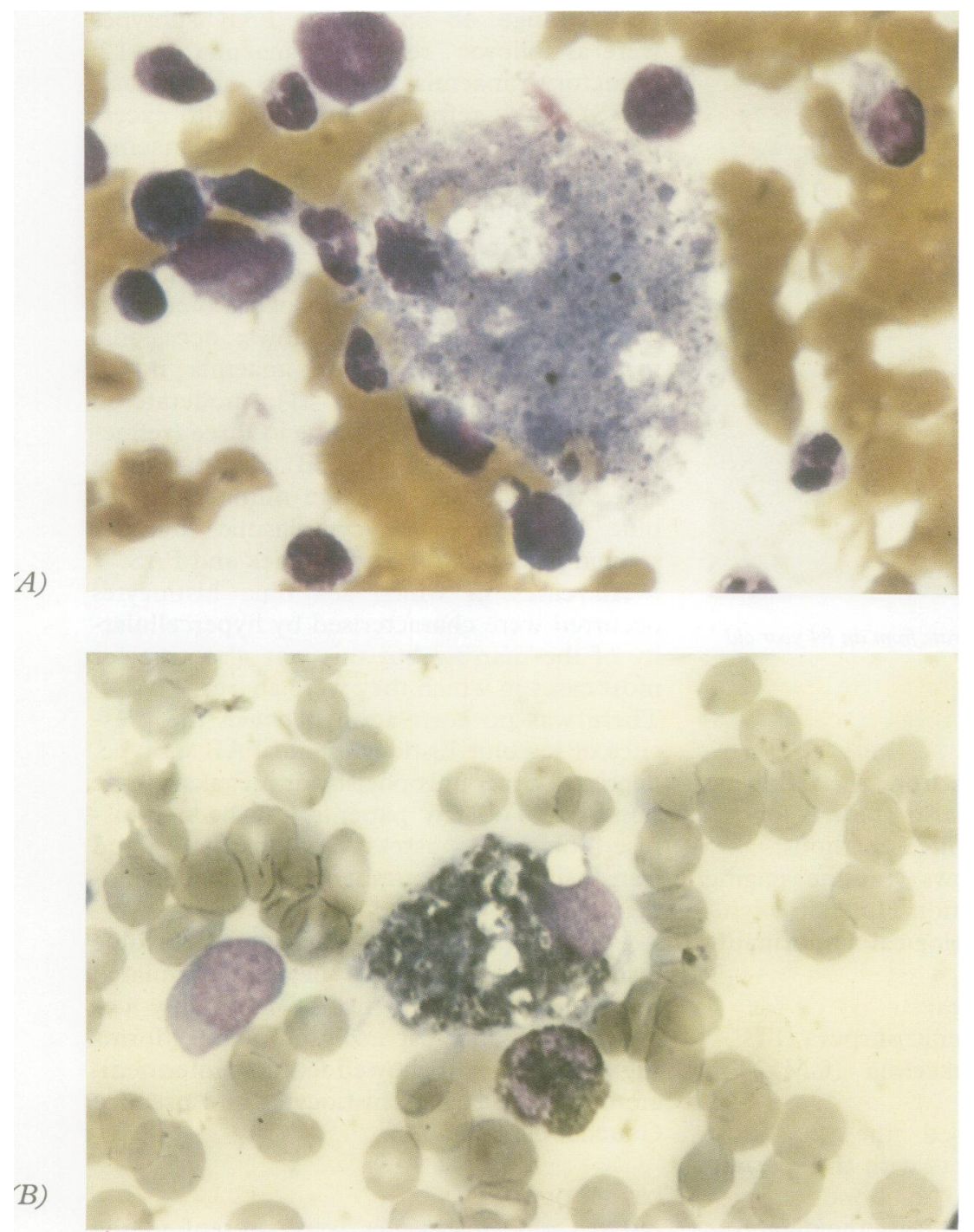

Figure $3 A$ Sea blue histiocyte in the marrow of a 65 year old man with refractory anaemia. The cell has features intermediate between a classic sea blue histiocyte and a pseudo-Gaucher cell. B Sea blue histiocyte from the same case stained with Sudan black.

There was no correlation with any other morphological characteristics or FAB subtype. Bone marrow aspirates were performed at presentation and too few patients had been transfused to assess the effect of blood transfusion on numbers of sea blue histiocytes. Variation in the degree of cytoplasmic granulation was also noted by Silverstein et $\mathrm{al}^{3}$ in their original description of the "sea blue histiocyte syndrome". Kelsey et al noted storage cells with features intermediate between classic sea blue histiocytes and pseudo-Gaucher cells in their study of CML. ${ }^{13}$

The staining characteristics of sea blue histiocytes in MDS are consistent with the view that they are ceroid-containing macrophages. ${ }^{7}$ It has been suggested that accumulation of these cells in the marrow is a secondary phenomenon associated with excessive turnover of haematopoietic cells. ${ }^{6}$ Under these circumstances macrophages may be unable to metabolise completely the lipids of phagocytosed blood cells. Sea blue histiocytes probably arise in MDS as a result of ineffective haematopoiesis leading to increased destruc- tion of erythrocytes, leucocytes, and platelets and resultant saturation of normal pathways for removal of membrane lipid. Sea blue histiocytes were not found in the hypercellular marrows of polycythaemia, suggesting that it is the extent of ineffective haematopoiesis rather than the absolute degree of marrow hypercellularity which is the key factor. This hypothesis is further supported by a report of bone marrow sea blue histiocytes in thalassaemia. ${ }^{14}$ Thalassaemia was excluded from this study as there is not a suitable population of such patients in this region of the United Kingdom.

There may be other mechanisms causing sea blue histiocytes to form in MDS. For example, macrophages belonging to the preleukaemic clone could be functionally abnormal with defective catabolic enzymes. The formation of sea blue histiocytes would thus represent either the abnormal macrophages or remaining normal macrophages overwhelmed by the lipid load. Future prospective studies of sea blue histiocytes in MDS might include electron microscopy and, in patients with cytogenetic abnormalities, in situ hybridisation with chromosome specific probes to determine the cells' clonal nature.

In conclusion, sea blue histiocytes are a common cytological feature of the bone marrow of patients with MDS. As the other disorders documented as being frequently associated with bone marrow sea blue histiocytes occur relatively rarely myelodysplasia is probably the most common cause of this phenomenon, at least in a northern European population.

We thank Mrs Pam McCardle for her technical assistance

1 Wewalka F. Zur frage der blauen pigmentmakrophagen und sternalpunktat. Wien Klin Wochenschr 1950;62. und sterna $788-91$.

2 Varela-Duran J, Roholt PC, Ratliff NB. Sea-blue histiocyte syndrome. A secondary degenerative process of macrophages? Arch Pathol Lab Med 1980;104:30-4

3 Silverstein MN, Ellefson RD, Athern EJ. The syndrome of the sea-blue histiocyte. N Engl F Med 1970;282:1-4.

4 Dosik H, Rosner F, Sawitsky A. Acquired lipidosis: Gaucher like cells and blue cells in chronic granulocytic leukaemia. Semin Haematol 1972;9:309-16.

5 Heyhoe FGJ, Flemens RJ. A colour atlas of haematological cytology. London: Wolfe Medical Publications Ltd, cytology. $1982: 169$.

6 Kattlove HE, Gaynor E, Spivack M, Gottfried EL. Sea blue indigestion. $N$ Engl $\mathcal{F}$ Med 1970;282:630-1. 7 Rywlin AM, Hernandez JA, Chastain DE, Pardo V. pathic thrombocytopenic purpura (ITP): A contribution to the understanding of the sea blue histiocyte. Blood 1971;37:587-93.

8 Krause JR, Lee RE. Histiocytic infiltrates. In: Krause JR, ed. Bone marrow biopsy. New York: Churchill Livingstone, 1981:177.

9 Wickramasinghe $\mathrm{SN}$, ed. Blood and bone marrow. In: Systemic pathology. Vol 2. Edinburgh: Churchill Livingstone, 1986:88-9.

10 Hoffbrand AV, Petit JE. Sandoz atlas. In: Clinical haematology. London: Gower, 1988:263.

11 Bain BJ, Clark DM, Lampert IA. Miscellaneous disorders. In: Bone marrow pathology. Oxford: Blackwell Scientific

12 Bennett JM, Catovsky D, Daniel MT, et al. Proposals for the classification of the myelodysplastic syndromes. $\mathrm{Br} \mathcal{J}$ Haematol 1982;51:189-99.

13 Kelsey PR, Geary CG. Sea blue histiocytes and Gaucher cells in bone marrow of patients with chronic myeloid leukaemia. $f$ Clin Pathol 1988;41:960-2.

14 Beltrami CA, Bearzi I, Fabris G. Storage cells of spleen and bone marrow in thalassaemia: An ultrastructural study. Blood 1973;41:901-12. 